\title{
Concepções de estudantes do Ensino Médio sobre os conceitos de média e dispersão de dados ${ }^{+*}$
}

\author{
Alessandro Damásio Trani Gomes ${ }^{1}$ \\ Departamento de Ciências Naturais \\ Universidade Federal de São João del-Rei \\ São João del-Rei - MG
}

\section{Resumo}

Este trabalho investiga como um grupo de estudantes do Ensino Médio lida com dados no laboratório de Física. Em particular, como eles representam o melhor valor de uma medida e quais significados eles atribuem ao cálculo do valor médio como uma das formas de se apresentar o valor mais representativo de um conjunto de dados experimentais. Avalia também a evolução na capacidade dos estudantes em julgar a qualidade dos dados, levando-se em consideração a dispersão dos valores dos conjuntos. Ao todo, 250 estudantes do $1^{\circ}$ ano do Ensino Médio participaram de pelo menos uma das etapas da pesquisa, que consistiu na aplicação de dois testes, um no início e outro no final do ano letivo. A comparação dos resultados do pré e do pós-teste indica, para o grupo de alunos pesquisado, certa sofisticação na forma de representação de dados experimentais e acerca dos significados atribuídos aos construtos relacionados. Com base nos resultados obtidos, são discutidas as implicações educacionais e propostas novas possibilidades de pesquisa na área.

Palavras-chave: Ensino de Física; Atividades experimentais; Análise de dados.

\footnotetext{
Abstract

This work investigates how a group of secondary school students deals with sets of experimental data in the Physics laboratory. In particular, it

' Secondary school students' conception about the concepts of average and data dispersion

* Recebido: junho de 2015. Aceito: novembro de 2015.

${ }^{1}$ E-mail: alessandrogomes@ufsj.edu.br
} 
focuses on how the students represent the best value of a physical quantity through a set of repeated measurements, and the meanings they take into account for the computation of the average value. The paper also examines the development of students' ability to judge the quality of data, considering the data dispersion. In total, 250 students from the 1st year of high school participated in at least one of the phases of the research, which consisted in the application of two tests, one at the beginning and one at the end of the school year. For this group of students, a certain level of sophistication was identified on their representations of experimental data and their understanding of the related constructs. Based on these results, we discuss the educational implications and propose new possibilities for research in the area.

Keywords: Physics teaching; Laboratory activities; Data analysis.

\section{Introdução}

Existe hoje uma enorme diferença quanto às atividades e atuações das pessoas envolvidas na comunidade científica. Mas algumas, em especial, merecem grande destaque, sobretudo nas Ciências Naturais. As atividades de coleta, análise e interpretação de dados estão presentes em grande parte da atividade científica e fundamentam toda pesquisa baseada em dados experimentais. Quando se fala em coletar dados e avaliar evidências, há, ainda, uma ação mais básica que perpassa por todos os estudos científicos quantitativos: a mensuração. Efetuar uma medida é o ato de quantificar um determinado atributo do sistema. Tal ato é baseado na interação entre o ente físico do sistema que se deseja medir e o instrumento que é utilizado para efetuar a medida.

$\mathrm{Na}$ Ciência, dados são utilizados para avaliar hipóteses e/ou responder a problemas de pesquisa. Engenheiros utilizam tabelas e gráficos de medidas para tomar decisões quanto aos projetos. Economistas e cientistas sociais utilizam medidas para descobrir tendências e fazerem previsões. A utilização de dados científicos não é limitada a pesquisadores, técnicos e engenheiros. O público em geral também é responsável por interpretar informações científicas e tomar decisões baseadas em resultados de estudos experimentais. A qualidade de todas as decisões baseadas em dados depende, em grande parte, de dois componentes: a qualidade das medidas efetuadas e da estimativa da incerteza sobre esses dados (LIKHACHEV; CRUZ; MESA, 2000).

Desde a década de 1990, educadores reconhecem o papel do processo de mensuração e dos conceitos a ele relacionados no Ensino de Ciências. Além disso, a importância de uma boa compreensão sobre o processo de medição vem sendo destacada em documentos curriculares oficiais em diversos países, inclusive no Brasil (AAAS, 1990; BRASIL, 1999, NRC, 2000). 
Apesar de ser fundamental para o desenvolvimento da Ciência e para a formação de uma sociedade crítica, é relativamente pequena a atenção que é dada, na pesquisa em Educação em Ciências, às concepções e habilidades dos estudantes envolvidas na coleta e interpretação dos dados e de seus conceitos adjacentes. As pesquisas na área (KUNG; LINDER, 2006; MARINELLI; PACCA, 2006; LOVETT; SHAH, 2007; LABURÚ; SILVA; SALES, 2010; LUBBEN; ALLIE; BUFFLER, 2010; MUNIER; MERLE; BREHELIN, 2013, por exemplo) apontam que estudantes, em todos os níveis de ensino, possuem grandes dificuldades com relação aos conceitos e conhecimentos envolvidos no processo de medição e análise das medidas produzidas.

O laboratório de Ciências, especialmente o de Física, pode fornecer a oportunidade para os estudantes aprenderem sobre o processo de medição e coleta de dados. Na maioria das atividades práticas desenvolvidas nos laboratórios, os estudantes efetuam medidas constantemente, avaliam e analisam os dados obtidos. Os dados são utilizados para calcular outras grandezas seja para testar, seja para formular hipóteses. Questões sobre como representar uma medida e sobre a qualidade dos dados obtidos parecem surgir naturalmente, o que levaria os estudantes a desenvolverem uma compreensão mais sofisticada, isto é, mais próxima daquilo que é aceito pela comunidade científica acerca do processo de medição, bem como da análise e tratamento dos dados.

Quando um mesmo experimentador executa diversas medidas de uma grandeza física, sob as mesmas condições, ele obtém um conjunto de medidas que apresentarão valores diferentes devido aos erros experimentais inerentes ao processo. Helene, Tsai e Teixeira (1991), Vuolo (1996) e Likhachev, Cruz e Mesa (2000) argumentam que se os valores medidos forem estatisticamente independentes e obedecerem à distribuição normal, ou de Gauss (o que ocorre em praticamente todas as atividades experimentais no Ensino Médio), o valor que mais se aproxima do valor verdadeiro ou valor alvo da grandeza experimental é a média aritmética dos valores medidos, denominado valor médio. Espera-se, ainda, que o valor médio calculado seja tanto mais preciso e exato quanto maior for o número de medidas. Além disso, para que a medida esteja adequadamente descrita, segundo Helene, Tsai e Teixeira (1991), é necessário também apresentar "uma estimativa do quão longe o valor verdadeiro pode estar da estimativa que temos dele" (p.15), que pode ser, em boa parte dos casos, o desvio padrão da média.

É importante ressaltar que o valor médio não é um estimador universalmente indicado como o valor que melhor representa qualquer conjunto de dados. Devemos ficar atentos a contextos e casos nos quais isso ocorre. A forma da distribuição do conjunto influencia na escolha correta de uma medida de tendência central. Segundo Levin e Fox (2004), por exemplo, a mediana é a medida de tendência central mais conveniente para descrever um conjunto de dados com uma distribuição muito assimétrica de valores. Isso ocorre porque a mediana se situa sempre entre a média e a moda e, ao contrário da média, a mediana é menos suscetível à presença ou à mudança de valores extremos no conjunto. 
Há, ainda, diversos fenômenos naturais e do cotidiano que não são apropriadamente descritos por meio da distribuição normal. O número de partículas alfa emitidas pelo decaimento de uma amostra de material radioativo por unidade de tempo, o número de clientes que entram em um supermercado em sua primeira hora de funcionamento ou a quantidade de peças defeituosas produzidas por uma fábrica por dia, são exemplos de fenômenos que ocorrem aleatoriamente, mas, a uma taxa média definida. Tais fenômenos são comumente modelados utilizando-se a distribuição de probabilidades de Poisson, para a qual as medidas de tendência central tradicionais possuem pouco ou nenhum significado.

As análises que são apresentadas neste trabalho, fazem parte de um projeto de pesquisa mais amplo que busca investigar as possíveis aprendizagens decorrentes da realização de atividades práticas no laboratório de Física durante o Ensino Médio. Tendo esse contexto em vista e diante do exposto, o objetivo do artigo é descrever e analisar a importância e os significados que um grupo de estudantes atribui ao cálculo da média no contexto da experimentação e a importância que este grupo atribui à dispersão dos dados para a avaliação e comparação da qualidade dos dados experimentais.

Sendo assim, busca-se, nesse trabalho, responder a três questões:

1) Os estudantes do grupo pesquisado compreendem a importância e o significado do cálculo da média para a determinação do valor mais representativo de conjuntos de dados experimentais no contexto de atividades práticas do Ensino Médio?

2) Os estudantes conseguem avaliar a qualidade de um conjunto de dados experimentais levando-se em consideração a dispersão dos valores do conjunto?

3) Como essas compreensões se desenvolvem ao longo de um ano letivo, após a realização de uma sequência de atividades experimentais?

\section{Paradigma pontual e paradigma de conjunto}

Qualquer processo de medida, quando repetido sistematicamente, produz uma série de valores e, para se analisar tais resultados, é preciso uma compreensão sobre os limites desses valores, a dispersão dos dados obtidos e também, sobre as causas dessa variabilidade. A interpretação dos resultados depende, portanto, de uma estimativa numérica da grandeza medida e da avaliação da dispersão dos dados (HELENE; TSAI; TEIXEIRA, 1991), e também, das considerações sobre as possíveis fontes de erros e perturbações provocadas no sistema.

Fred Lubben e membros do seu grupo de pesquisa (ALLIE et al., 1998; BUFFLER et al., 2001; LUBBEN et al., 2001), realizaram uma série de trabalhos que forneceu um importante suporte empírico para o desenvolvimento de um referencial no qual o pensamento e o raciocínio utilizados pelos estudantes durante o processo de medição e análise dos dados podem ser categorizados em dois tipos distintos, segundo os paradigmas Pontual e de Conjunto ${ }^{2}$. As diferenças entre os paradigmas podem ser vistas no Quadro 1.

\footnotetext{
2 Tradução dos termos em inglês point e set paradigms, conforme Laburú e Barros (2009).
} 
Quadro 1 - Caracterização dos paradigmas Pontual e de Conjunto.

\begin{tabular}{|l|l|}
\hline \multicolumn{1}{|c|}{ Paradigma Pontual } & \multicolumn{1}{c|}{ Paradigma de Conjunto } \\
\hline $\begin{array}{l}\text { O processo de medição permite determinar o } \\
\text { valor verdadeiro da grandeza. }\end{array}$ & $\begin{array}{l}\text { O processo de medição fornece informações } \\
\text { incompletas sobre a grandeza que está sendo } \\
\text { medida. }\end{array}$ \\
\hline $\begin{array}{l}\text { "Erros" acontecem devido a uma inabilidade } \\
\text { do experimentador ou devido às falhas na } \\
\text { configuração experimental. }\end{array}$ & $\begin{array}{l}\text { Todas as medidas estão sujeitas a incertezas } \\
\text { que não podem ser eliminadas. As incertezas } \\
\text { podem apenas ser reduzidas. }\end{array}$ \\
\hline $\begin{array}{l}\text { Uma única medida tem o potencial de ser o } \\
\text { valor verdadeiro da grandeza que está sendo } \\
\text { medida. }\end{array}$ & $\begin{array}{l}\text { As medidas realizadas são utilizadas para } \\
\text { construir uma distribuição a partir da qual a } \\
\text { melhor aproximação da grandeza medida e a } \\
\text { dispersão dos dados serão obtidas. }\end{array}$ \\
\hline
\end{tabular}

Fonte: traduzido de Lubben; Allie; Buffler (2010, p. 137).

O paradigma Pontual se caracteriza pela noção de que cada medida pode, em princípio, ser o valor correto. Como consequência, cada medida é independente das outras e as medidas individuais não são combinadas de forma alguma com as outras. Portanto, cada medida é concebida como sendo um valor, ao invés de contribuir para se estabelecer um intervalo. $\mathrm{Na}$ forma mais extrema do pensamento, segundo esse paradigma, está a crença de que apenas uma medida é necessária para estabelecer o valor verdadeiro da grandeza medida.

O paradigma de Conjunto é caracterizado pela ideia de que cada medida é apenas uma aproximação do valor verdadeiro e que o desvio deste valor é aleatório. Como consequência, um número de medidas é necessário para formar uma distribuição que se estabeleça em torno de um valor determinado. A melhor informação sobre o valor verdadeiro é obtida pela combinação das medidas, utilizando-se construtos teóricos para descrever os dados conjuntamente.

Como parte do seu desenvolvimento e compreensão dos métodos utilizados pela Ciência para obtenção de evidências válidas, os indivíduos devem aprender procedimentos adequados de análise de dados (ações) e aprofundar sua compreensão sobre a natureza dos dados obtidos e do processo de medição (raciocínio). Suas ações e raciocínios também podem ser associados aos paradigmas Pontual e de Conjunto como ilustra o Quadro 2.

A principal diferença entre os dois paradigmas é que os estudantes que se orientam segundo o paradigma Pontual tiram suas conclusões e inferências a partir dos pontos ou dados isolados, enquanto quem se orienta segundo o paradigma de Conjunto, considera todo o conjunto dos dados disponíveis para tal. 
Quadro 2 - As ações e raciocínios dos indivíduos durante o processo de experimentação.

\begin{tabular}{|c|c|c|}
\hline $\begin{array}{l}\text { Fase do processo } \\
\text { de medição }\end{array}$ & Ações & Raciocínios \\
\hline \multicolumn{3}{|c|}{ Paradigma Pontual } \\
\hline Coleta de dados & $\begin{array}{l}\text { A repetição de medidas não é neces- } \\
\text { sária, ou é feita para achar o mesmo } \\
\text { valor ou repetir por prática. }\end{array}$ & $\begin{array}{l}\text { Uma medida nos conduz a um } \\
\text { ponto. Apenas uma boa medida é } \\
\text { necessária. }\end{array}$ \\
\hline $\begin{array}{l}\text { Processamento } \\
\text { de dados }\end{array}$ & $\begin{array}{l}\text { Uma única (melhor) medida é esco- } \\
\text { lhida (moda) para representar a me- } \\
\text { dida. }\end{array}$ & $\begin{array}{l}\text { Cada medida é independente das } \\
\text { outras e pode ser, a princípio, o va- } \\
\text { lor verdadeiro. }\end{array}$ \\
\hline Gráficos & $\begin{array}{l}\text { Todos os pontos são unidos por seg- } \\
\text { mentos múltiplos de reta. }\end{array}$ & $\begin{array}{l}\text { O comportamento dos dados é me- } \\
\text { lhor representado por meio da es- } \\
\text { colha de determinados pontos que } \\
\text { descrevem o comportamento espe- } \\
\text { rado. }\end{array}$ \\
\hline $\begin{array}{c}\text { Comparando } \\
\text { um conjunto de } \\
\text { dados }\end{array}$ & $\begin{array}{l}\text { Uma comparação valor a valor é re- } \\
\text { alizada ou compara-se a média } \\
\text { quando esta é fornecida. }\end{array}$ & $\begin{array}{l}\text { A comparação é feita por meio da } \\
\text { avaliação de proximidade entre os } \\
\text { valores. }\end{array}$ \\
\hline \multicolumn{3}{|c|}{ Paradigma de Conjunto } \\
\hline Coleta de dados & $\begin{array}{l}\text { A repetição de medidas é necessária } \\
\text { como consequência das variações } \\
\text { possíveis. }\end{array}$ & $\begin{array}{l}\text { Cada medida é apenas uma aproxi- } \\
\text { mação do valor real e os desvios } \\
\text { produzidos são aleatórios. Um } \\
\text { grande número de medidas é ne- } \\
\text { cessário para formar uma distribui- } \\
\text { ção próxima do valor procurado. }\end{array}$ \\
\hline $\begin{array}{l}\text { Processamento } \\
\text { de dados }\end{array}$ & $\begin{array}{l}\text { Um conjunto de dados é represen- } \\
\text { tado pela média e seu desvio padrão. }\end{array}$ & $\begin{array}{l}\text { A melhor representação de uma } \\
\text { medida é obtida por meio da com- } \\
\text { binação das medidas utilizando } \\
\text { construtos teóricos como a média, } \\
\text { para caracterizar o conjunto de da- } \\
\text { dos como um todo. }\end{array}$ \\
\hline Gráficos & $\begin{array}{l}\text { A melhor curva é ajustada entre os } \\
\text { pontos disponíveis. }\end{array}$ & $\begin{array}{l}\text { A melhor representação gráfica de } \\
\text { uma série de medidas é o ajuste de } \\
\text { uma curva sobre os pontos dispo- } \\
\text { níveis. }\end{array}$ \\
\hline $\begin{array}{l}\text { Qualidade dos } \\
\text { dados }\end{array}$ & $\begin{array}{l}\text { Para um mesmo conjunto de dados, } \\
\text { o melhor será aquele que apresentar } \\
\text { o menor desvio padrão. }\end{array}$ & $\begin{array}{l}\text { O desvio padrão está relacionado } \\
\text { com a precisão da medida reali- } \\
\text { zada. }\end{array}$ \\
\hline $\begin{array}{l}\text { Comparando } \\
\text { um conjunto de } \\
\text { dados }\end{array}$ & $\begin{array}{l}\text { A comparação ocorre levando em } \\
\text { consideração a média e a sobreposi- } \\
\text { ção das dispersões dos dados. }\end{array}$ & $\begin{array}{l}\text { A média e o desvio padrão definem } \\
\text { o intervalo de confiança, que de- } \\
\text { fine o melhor valor para a medida } \\
\text { e a sua confiabilidade. }\end{array}$ \\
\hline
\end{tabular}

Fonte: traduzido e adaptado de Buffler et al., 2001. 
A utilização inconsistente dos paradigmas Pontual e de Conjunto por um mesmo indivíduo foi identificada nos trabalhos de Allie et al. (2001) e Buffler et al. (2001). Segundo os autores, é comum um indivíduo que se orienta segundo o paradigma Pontual, reconhecer, em determinado contexto, a necessidade de se calcular a média dos dados obtidos. Além disso, os autores perceberam que falta a muitos indivíduos que demonstram se orientar pelo paradigma de Conjunto uma compreensão profunda e completa dos conceitos que estão envolvidos no processo de medição e análise dos dados. Como exemplo, os autores citam estudantes que afirmam que repetem as medidas para poder calcular a média, mas escolhem outro valor para representar o conjunto de dados. Essas contradições acontecem com certa frequência em todas as fases do processo de medição. Tal fato corrobora as evidências de pesquisas anteriores que identificam que o desempenho de estudantes em atividades práticas é influenciado pelas demandas conceituais e pelo contexto da atividade (LEACH et al., 2000; MARINELI; PACCA, 2006).

Esse referencial será utilizado para se analisar a evolução das concepções dos estudantes sobre a importância e o significado do cálculo da média para a determinação do valor mais representativo de conjunto de dados experimentais e também para avaliar a evolução na capacidade dos estudantes em julgar a qualidade de um conjunto de dados experimentais, levandose em consideração a dispersão dos dados.

\section{Metodologia da pesquisa}

Para avaliar as aprendizagens e a importância pedagógica das diversas atividades realizadas ao longo de todo ano letivo, fez-se necessário um estudo que se utiliza da reaplicação dos instrumentos de pesquisa. Em um estudo longitudinal, devem-se ter duas preocupações básicas. A primeira é a adoção de um espaçamento adequado entre as coletas de dados para permitir que as mudanças e os conhecimentos adquiridos pelos indivíduos possam ser assimilados e incorporados. A segunda é a utilização, nas coletas de dados que podem ocorrer, de instrumentos semelhantes, passíveis de comparação entre si (WHITE; ARZI, 2005).

\section{III.1 Sujeitos da pesquisa}

A coleta de dados ocorreu em uma escola de Ensino Médio da rede federal de ensino de Belo Horizonte. Dos 261 estudantes matriculados no $1^{\circ}$ ano, $250^{3}$ participaram de pelo menos uma das etapas de coleta de dados da pesquisa. Os estudantes eram divididos em oito turmas com trinta a trinta e quatro alunos cada. Quatro professores de Física eram responsáveis, cada um, por duas turmas.

\footnotetext{
3 Ao todo, 238 alunos responderam o pré-teste e 173 o pós-teste. Não foi possível aplicar o pós-teste em duas turmas por questão de disponibilidade de datas.
} 
Optou-se por trabalhar com alunos do $1^{\circ}$ ano do Ensino Médio principalmente porque, por serem do $1^{\circ}$ ano, a experiência dos alunos em atividades de laboratório é limitada, uma vez que o contato, no Ensino Fundamental, com atividades em laboratório não é comum na maioria das escolas brasileiras.

Nessa escola, o currículo de Física tem uma componente experimental, aproveitandose da estrutura de laboratórios e os recursos disponíveis. Os alunos realizam atividades práticas quinzenalmente. As turmas são subdivididas em duas subturmas cada, com aproximadamente quinze estudantes. A cada semana, uma subturma realiza as atividades propostas.

Ao longo do ano letivo, foram desenvolvidas 13 atividades no laboratório de Física, todas integradas ao currículo normal de Física do $1^{\circ}$ ano da escola. As atividades envolveram conceitos de mecânica (movimento uniforme e variado, energia, sistema massa-mola e força elástica) e eletricidade (circuitos simples com pilhas e lâmpadas e medição de corrente e tensão em circuitos série e paralelo). As atividades possuíam diversos níveis de abertura (BORGES, 2002) e tinham diferentes objetivos, entre eles: aprender a formular e explicitar suas hipóteses, aprender como comunicar os resultados da atividade (através de relatórios), aprender como processar, representar e analisar dados.

É importante ressaltar que os alunos tiveram, no início do ano, três aulas teóricas (50 minutos cada) sobre teoria de erros, tipologia de erros e cálculo do valor médio e desvio absoluto médio. Além disso, também fizeram uma atividade especialmente planejada para trabalhar diretamente esses conceitos relativos ao processo de medição. Nas demais atividades práticas do ano, os alunos calcularam a média de conjuntos de dados, mas questões sobre variabilidade e a dispersão de dados experimentais não foram abordadas de forma explícita.

\section{2 Instrumentos de pesquisa}

Para identificar se os estudantes reconhecem a importância e o sentido de se determinar o valor médio, utilizou-se a Questão 1 em que são apresentados os dados em uma tabela de uma suposta atividade no laboratório de Física realizada por um grupo de alunos que mediu o tempo que uma esfera gasta para rolar sobre uma rampa (Fig. 1). É solicitado, então, que o aluno escreva qual o valor que melhor representa o tempo que a esfera gasta para percorrer a rampa e apresente uma justificativa.

Uma segunda questão foi elaborada para esclarecer melhor as concepções dos estudantes sobre o conceito de média e sua importância. A Questão 2, assim formulada - Em várias atividades no laboratório você determinou a média de um conjunto de valores. O que representa a média? O que ela significa? Por que é importante calculá-la? - foi aplicada apenas no pós-teste.

Para analisar melhor a visão dos estudantes sobre a variabilidade e dispersão dos dados e a importância atribuída à média ao se comparar dois conjuntos de dados, utilizou-se a Questão 3 que narra a história de dois grupos que, durante uma aula de laboratório, mediram o tempo 
que uma esfera gasta para percorrer uma rampa (Fig. 2). Os grupos A e B realizaram seis medidas de tempo cada um e estes dados são apresentados numa tabela, com sua respectiva média ao lado. Os dois conjuntos apresentam a mesma média, porém, a dispersão dos dados de um dos grupos é maior do que o do outro.

\section{Questão 1}

Durante a realização de uma atividade no laboratório de Física, um grupo de alunos mediu o tempo que uma esfera gasta para percorrer uma rampa. Todas as medidas foram realizadas com a mesma esfera e soltando-a de uma mesma altura. Os valores estão representados na tabela abaixo:

\begin{tabular}{|l|l|l|l|l|l|l|l|}
\hline Tempo (s) & 1,5 & 1,3 & 1,5 & 1,2 & 1,7 & 1,5 & 1,1 \\
\hline
\end{tabular}

De acordo com os dados na tabela, escreva qual o valor que melhor representa o tempo que a esfera gasta para percorrer a rampa. Justifique sua resposta.

Fig. 1 -Questão sobre o valor mais representativo de um conjunto de dados.

\section{Questão 3}

Durante a realização de uma atividade no laboratório de Física, dois grupos de alunos mediram o tempo que uma esfera gasta para percorrer uma rampa. Todas as medidas foram realizadas com esferas de mesma massa e soltando-as de uma mesma altura. Os valores estão representados na tabela abaixo:

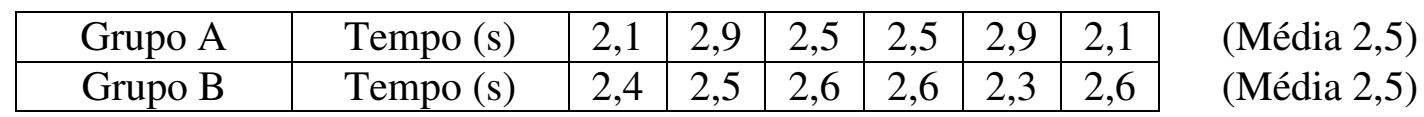

Sobre os dados, podemos fazer os seguintes comentários:

Comentário 1 - Os dados do grupo A são melhores, pois eles obtiveram dois valores iguais à média.

Comentário 2 - Os dados do grupo B são melhores, pois as medidas estão todas entre 2,3 e 2,6 s, valores mais próximos da média.

Comentário 3 - Os dados do grupo A são tão bons quanto os dados do grupo B, pois ambos possuem a mesma média.

Qual dos comentários você concorda mais? Justifique sua resposta.

Fig. 2 - Questão sobre a comparação entre dois conjuntos de dados.

Em seguida, são apresentados três comentários. Os alunos foram orientados a escolher que comentário eles julgavam mais apropriado e a fornecer uma justificativa para a escolha. $\mathrm{O}$ objetivo nessa questão é analisar como os estudantes comparam dois conjuntos de dados. $\mathrm{O}$ 
interesse é saber se os estudantes observam apenas os valores da média dos conjuntos ou se eles percebem a diferença quanto à dispersão dos dados dos conjuntos, ou seja, se os estudantes reconhecem que a dispersão também é um indicador da qualidade dos dados coletados, pois, quanto maior for essa dispersão, maior o desvio padrão do conjunto de dados, diminuindo-se assim, a precisão da medida. Versões modificadas dessas questões podem ser encontradas em outras pesquisas (LEACH et al., 2000; LUBBEN et al., 2001).

\section{III.3 Procedimentos}

Os dados para este trabalho foram coletados no início do ano, para se identificar os conhecimentos iniciais dos participantes e também, ao final do ano letivo, para que se pudessem identificar as possíveis modificações nesses conhecimentos, constituindo dessa forma um préteste e um pós-teste. Tanto no início, quanto no final do ano, a coleta do material empírico da pesquisa ocorreu durante o horário das aulas de Física dos estudantes (teóricas ou no laboratório), sob a responsabilidade dos próprios professores de Física das turmas. Tais professores possuem experiência e são pesquisadores em Ensino de Física.

Para cada questão, foi elaborada uma folha de resposta adequada. As questões, tanto no pré, quanto no pós-teste, foram aplicadas em dias distintos durante o horário normal de aula. Todos os alunos participantes e seus responsáveis assinaram o termo de consentimento livre e esclarecido antes da realização da pesquisa. Antes da aplicação, os professores novamente reforçavam a importância da participação dos estudantes e esclareciam os objetivos gerais da pesquisa.

Além da realização do pré-teste e do pós-teste, foram feitas entrevistas com um total de 25 estudantes (selecionados de acordo com a disponibilidade e voluntariedade), em grupo e/ou individualmente para esclarecer melhor suas concepções e ideias sobre o assunto e discutir melhor as respostas apresentadas nos testes. As entrevistas realizadas foram registradas em áudio e vídeo. Os cadernos de relatórios dos alunos de duas turmas (cerca de 70 cadernos), contendo o relatório produzido para as 13 atividades desenvolvidas ao longo de todo período letivo também foram recolhidos para análise.

\section{Análise dos dados}

Ao realizar uma atividade prática o estudante precisa, inicialmente, definir quais as variáveis devem ser observadas e medidas, possuir habilidades básicas para lidar com os instrumentos de medida, definir quais e quantos dados serão coletados e a maneira e estratégia mais adequada para coletá-los. Após os dados serem coletados, eles devem ser analisados. O primeiro passo para a análise dos dados, na grande maioria dos casos, é o cálculo de uma medida de tendência central do conjunto que, normalmente, é o valor médio.

Analisou-se o conjunto de respostas que os alunos forneceram à Questão 1 e pôde-se identificar três categorias de respostas recorrentes: 
M1 - Média: Os estudantes responderam que o valor que melhor representava o conjunto de dados é a média. Na maioria dos casos, eles calcularam a média. Exemplos:

O melhor resultado é 1,4 s já que este resultado representa a média dos outros.

O melhor número é 1,4, pois representa a média de todos os números que apareceram.

M2 - O valor da tabela que mais se repetiu: Nesse tipo de resposta, o estudante afirmou que o valor que melhor representa o conjunto de medições é a moda do conjunto, ou seja, o valor que mais se repete. Neste caso, afirmaram que o valor seria 1,5 segundos. Exemplos:

Eu acho que o valor que melhor corresponde esse tempo é 1,5s pois ele aparece mais de uma vez na tabela.

O valor de número 1,5. Pois por ser a mesma esfera e uma mesma altura, esse valor se repete frequentemente, representando melhor o tempo para que a esfera percorra a rampa.

M3 - Calcula a média e escolhe outro valor: Alguns alunos calcularam a média e, após o cálculo, escolheram algum valor presente na tabela que mais se aproximasse da média. Nesse caso, os valores escolhidos foram 1,3 e/ou 1,5 segundos. Exemplos:

O valor mais próximo da média entre os resultados, pois assim, as chances de erro de medida diminuem. A média é $1,4 \mathrm{~s}$, portanto, $1,5 \mathrm{~s}$.

Seria 1,3 ou 1,5. Pois tirando a média o resultado seria 1,4 e estes são os que mais se aproximam.

A compreensão da importância da determinação do valor médio de um conjunto de dados também se relaciona com o conceito de reprodutibilidade. Um estudante que não prevê a variabilidade dos dados, não reconhece a importância, nem o sentido da média como o valor mais representativo de um conjunto de dados. Ao contrário, ele prefere escolher o valor que mais se repete dentro do conjunto (moda), pois acredita que os valores que diferem da moda estariam, supostamente, errados.

As categorias aqui desenvolvidas relacionam-se com os paradigmas propostos por Buffler et al. (2001). Pode-se associar a categoria M1 ao paradigma de Conjunto, pois o estudante reconhece a necessidade de se calcular a média dos valores do conjunto. A categoria M2 pode ser caracterizada segundo paradigma Pontual, pois o estudante observa individualmente os pontos da tabela. Já a categoria M3 pode ser indício de uma transição entre os paradigmas, pois o estudante calcula a média, ou seja, reconhece que os dados devem ser analisados em conjunto, mas escolhe um ponto do conjunto. Ela pode indicar também o entendimento equivocado da questão. O estudante que escolhe M3 pode ter entendido que era solicitado que ele escolhesse qual dentre aqueles valores tabelados melhor representa a medida. 
Na Tabela 1 está representada a distribuição de frequência das respostas dos estudantes, para o pré e pós-teste, de acordo com a categorização acima ${ }^{4}$. No pré-teste, 238 estudantes responderam à questão. Desses, mais da metade (51\%) tiveram suas respostas categorizadas como M1, reconhecendo a importância do cálculo da média para representar um conjunto de dados. Outros 89 estudantes (37\%) tiveram suas respostas categorizadas como M2. A categoria M3 e as respostas não categorizadas somaram apenas 11\%. É importante ressaltar que, quando os alunos realizaram o pré-teste, eles já haviam feito três aulas de laboratório e já haviam discutido em sala de aula questões sobre medidas e erros, cálculo de média, erros aleatórios, sistemáticos, e como representar a variabilidade dos dados através de desvios. Porém, mesmo após essas atividades (3 aulas e uma atividade de laboratório) específicas, quase a metade dos estudantes ainda não reconhece a necessidade do cálculo do valor médio para a representação de um conjunto de dados experimentais.

Tabela 1 - Distribuição das respostas dos estudantes para a Questão 1.

\begin{tabular}{ccccc}
\hline \multirow{2}{*}{ Categorias } & \multicolumn{2}{c}{ Pré-teste } & \multicolumn{2}{c}{ Pós-teste } \\
& Alunos & Percentual & $\begin{array}{c}\text { Alu- } \\
\text { nos }\end{array}$ & Percentual \\
\hline M1 & 122 & $51 \%$ & 117 & $68 \%$ \\
M2 & 89 & $37 \%$ & 37 & $21 \%$ \\
M3 & 13 & $6 \%$ & 13 & $8 \%$ \\
Outras & 14 & $6 \%$ & 6 & $3 \%$ \\
\hline Totais & $\mathbf{2 3 8}$ & $\mathbf{1 0 0} \%$ & $\mathbf{1 7 3}$ & $\mathbf{1 0 0} \%$ \\
\hline
\end{tabular}

No pós-teste, a questão foi respondida por 173 estudantes. Houve um aumento na percentagem de alunos que tiveram suas respostas categorizadas como M1 (68\%) e a diminuição da categoria M2 (21\%). As percentagens da categoria M3 e das respostas não categorizadas praticamente não se alteraram nos dois testes.

Para se comparar melhor os resultados dos testes, são apresentados os dados da Tabela $2^{5}$. Dos 166 estudantes que realizaram ambos os testes, 70 responderam corretamente nos dois testes (categoria M1). Dos 96 estudantes restantes, 41 (43\%) sofisticaram suas respostas no pós-teste, o que representou uma diferença significativa entre os resultados dos testes $\operatorname{MH}(166)=2,01, \mathrm{p}=0,05^{6}$.

\footnotetext{
${ }^{4}$ Devido à diferença no número de respondentes dos testes, serão apresentados os resultados de cada teste isoladamente e, em seguida, os resultados daqueles alunos que realizaram os dois testes.

${ }^{5}$ As células em destaque nas tabelas apresentam o número de alunos que sofisticaram suas concepções em relação ao pré-teste.

6 Para a verificação da significância das mudanças no entendimento dos participantes da pesquisa, foi utilizado o teste de homogeneidade marginal, que é similar ao teste de McNemar, porém, estendido, quando for o caso, para os casos nos quais a variável de interesse assume mais de dois valores nominais. Para cada teste realizado, reportase o valor padronizado da estatística do teste e o nível de significância obtido.
} 
Tabela 2 - Comparação dos resultados dos testes - Questão 1.

\begin{tabular}{ccccccc}
\hline & & \multicolumn{9}{c}{ Pós-teste } & & Total \\
& Categoria & M1 & M2 & M3 & Outras & \\
\hline \multirow{4}{*}{ Pré-teste } & M1 & 70 & 11 & 4 & 2 & $\mathbf{8 7}$ \\
& M2 & 32 & 19 & 6 & 3 & $\mathbf{6 0}$ \\
& M3 & 4 & 4 & 3 & 0 & $\mathbf{1 1}$ \\
& Outras & 5 & 2 & 0 & 1 & $\mathbf{8}$ \\
\hline \multicolumn{2}{c}{ Total } & $\mathbf{1 1 1}$ & $\mathbf{3 6}$ & $\mathbf{1 3}$ & $\mathbf{6}$ & $\mathbf{1 6 6}$ \\
\hline
\end{tabular}

Assim, 68\% dos estudantes que responderam a essa questão no pós-teste chegaram ao final do primeiro ano do Ensino Médio com uma compreensão adequada de que a média é o valor que, em muitos casos, melhor representa um conjunto de dados em uma atividade experimental. Esse percentual pode ser considerado, a princípio, como satisfatório. Em 10 atividades realizadas ao longo do ano letivo havia a necessidade de se calcular a média de algum conjunto de dados. Portanto, essa foi uma habilidade trabalhada repetidamente no laboratório, embora o tema só tenha sido abordado como conteúdo de ensino na primeira semana do ano letivo.

Nas entrevistas realizadas com os estudantes, perguntou-se aos estudantes sobre a importância do valor médio, o que ele representava e o porquê do seu cálculo. Para essas perguntas, poucos estudantes conseguiram articular respostas satisfatórias sobre o real significado da média e sua importância. A grande maioria das respostas foi insatisfatória, conforme exemplos a seguir:

A média é um resultado intermediário dos resultados obtidos.

Ela representa o valor mais apropriado de uma medição e é importante para tornar os resultados mais precisos.

É importante calcular a média, pois ela elimina os erros de medida.

As respostas acima indicam, assim como os resultados da Tabela 1, que a maioria dos estudantes reconheceu a necessidade de se calcular o valor médio das medidas obtidas. Porém, demonstram que não desenvolveram uma compreensão adequada sobre a importância do seu cálculo. Muitos argumentaram que o cálculo da média elimina os erros sem distinção, outros, dão respostas superficiais. Alguns estudantes, por outro lado, apresentaram respostas mais sofisticadas. Em algumas, parte dos estudantes reconhece que a média é uma aproximação do valor real e que seu cálculo pode eliminar apenas erros aleatórios de medida.

A média representa o valor mais apropriado. A média permite uma aproximação do valor real, podendo amenizar os erros aleatórios de medida. 
A média é um valor próximo ao valor real, pois ela elimina erros aleatórios.

Nos relatórios escritos pelos estudantes, também foi possível identificar algumas passagens que demonstram concepções incipientes sobre a importância do cálculo da média e também algumas passagens que demonstram certa sofisticação no pensamento:

É necessário medir três vezes para obter uma média, que funcionará como uma taxa de erro. Medindo três vezes há uma certeza maior para os resultados.

A partir desses dados, tiramos a média, pois ela minimiza os erros aleatórios e representa melhor a grandeza medida.

A partir das respostas às entrevistas ao longo do ano letivo, sentiu-se a necessidade de esclarecer melhor as compreensões dos estudantes participantes da pesquisa sobre o cálculo da média e sua importância durante uma atividade experimental. Para tanto, elaborou-se a Questão 2, que foi aplicada apenas no pós-teste.

Para análise das respostas a essa questão, foi desenvolvido um sistema de categorias que classifica as respostas dos estudantes quanto à sofisticação de suas concepções sobre a importância do cálculo do valor médio:

Sofisticada: Resposta na qual o aluno demonstrou compreender que o valor médio é o melhor valor para representar uma medida no contexto das atividades experimentais do Ensino Médio, pois o valor "exato" dessa medida é impossível de se determinar e que o cálculo da média reduz os efeitos de erros aleatórios. Exemplo:

Representa um valor que se procura, mas que devido aos erros de medida não conseguimos achar. É importante porque ao medirmos, os valores podem variar para mais ou para menos; a média ameniza essa diferença.

Suficiente: Resposta na qual o aluno comentou sobre quaisquer dois aspectos da resposta considerada Sofisticada. Exemplo:

A média representa o valor mais real, porque ao realizarmos as medidas cometemos alguns erros acidentais que ao fazer a média, reduzimos.

Insuficiente: Resposta na qual o estudante escreve sobre apenas um aspecto da resposta considerada Sofisticada e/ou comete pequenos equívocos na resposta à questão. A grande maioria das respostas assim classificadas, afirmavam que o cálculo da média aumentava a exatidão do experimento ou que reduzia/eliminava os erros de medida, indistintamente. Exemplos:

A média é melhor para expressar resultados, pois ela elimina os erros de medida que surgem. 
É o valor mais próximo da realidade. É importante porque significa maior precisão nas medidas.

Incipiente: Resposta vaga, baseada em uma concepção idiossincrática do estudante. Exemplo:

Ela é uma forma de termos valores mais certos, mais aproximados, porque dessa forma podemos simplificar bastante o que estamos fazendo.

Na Tabela 3 tem-se a distribuição das respostas dos estudantes de acordo com a categorização proposta. Os resultados corroboram as entrevistas e os dados da Tabela 1. Menos de $10 \%$ dos estudantes que responderam à questão desenvolveram, ao final do ano letivo, uma concepção adequada sobre a importância e o papel da média na análise de dados experimentais.

Os resultados até aqui apresentados nos deixam um alerta. Mesmo tendo realizado 13 práticas ao longo do ano letivo e, na grande maioria destas, os alunos terem calculado a média de diversos conjuntos de dados, parte dos estudantes que compõem o grupo pesquisado ainda não reconhece a necessidade de se calcular a média e a grande maioria deles (92\%) demonstrou dificuldades para atribuir ao valor médio o seu real significado e importância.

Tabela 3 - Distribuição das respostas dos estudantes para a Questão 2.

\begin{tabular}{ccc}
\hline Categorias & \multicolumn{2}{c}{ Pós-teste } \\
& Alunos & Percentual \\
\hline Sofisticada & 4 & $2 \%$ \\
Suficiente & 10 & $6 \%$ \\
Insuficiente & 113 & $70 \%$ \\
Incipiente & 35 & $22 \%$ \\
\hline Totais & $\mathbf{1 6 2}$ & $\mathbf{1 0 0 \%}$ \\
\hline
\end{tabular}

Analisou-se também as respostas dos estudantes para a Questão 3. Essa questão foi respondida por 202 estudantes no pré-teste e por 161 no pós-teste. Apresenta-se, na Tabela 4, a distribuição das percentagens de estudantes que optaram por um dos três comentários possíveis e pré-estabelecidos para a questão.

Tabela 4 - Número e percentagem para cada um dos comentários - Questão 3.

\begin{tabular}{ccccc}
\hline \multirow{2}{*}{ Comentários } & \multicolumn{2}{c}{ Pré-teste } & \multicolumn{2}{c}{ Pós-teste } \\
& Alunos & $\begin{array}{c}\text { Per- } \\
\text { centual }\end{array}$ & Alunos & Percentual \\
\hline Comentário 1 & 4 & $2 \%$ & 2 & $1 \%$ \\
Comentário 2 & 68 & $34 \%$ & 59 & $37 \%$ \\
Comentário 3 & 130 & $64 \%$ & 100 & $62 \%$ \\
\hline Totais & $\mathbf{2 0 2}$ & $\mathbf{1 0 0 \%}$ & $\mathbf{1 6 1}$ & $\mathbf{1 0 0 \%}$ \\
\hline
\end{tabular}


No pré-teste, a grande maioria dos estudantes optou pelo Comentário 3 e apenas 34\% dos alunos optaram pelo Comentário 2. No pós-teste, há um ligeiro aumento na percentagem de alunos que optaram pelo Comentário 2 (37\%) e, a consequente diminuição da percentagem do Comentário 3 (62\%). Nos dois testes, as percentagens dos alunos que optaram pelo Comentário 1 foram muito pequenas.

Percebe-se que, para a maioria dos alunos, apenas o valor médio importa para julgar a qualidade dos dados experimentais. Tal fato fica evidenciado pela análise das justificativas apresentadas:

Porque o melhor valor para uma medida de qualquer coisa é a média de vários valores, que permite um valor mais próximo do valor ideal. Se as médias dos dois grupos são iguais podemos ver como ambos obtiveram um mesmo valor quase ideal, ou seja, ambos são bons.

Comentário 3, pois com a mesma média eles possuem os mesmos erros e acertos.

Comentário 3 pois o valor da média é o mais importante na análise dos resultados do experimento.

Comentário 3. O importante é que eles chegaram à mesma média.

Porque ambos os grupos tiveram erros de medida. Não importa valores isolados, o que importa é a média, pois ela é o valor mais representativo das medidas. Como eles obtiveram a mesma média, os dois grupos estão corretos.

Nas entrevistas realizadas com os alunos, essa questão também foi abordada:

Tanto um quanto o outro são bons porque eles acharam a mesma média. Às vezes a gente pensa que o B é melhor porque teve uma variação menor. Mas acho que por terem obtido a mesma média os dois são bons.

Na minha opinião, se a média foi igual, acho que não tem diferença de qual é o melhor. O importante é a média.

Na Tabela 5 está representado o resultado da comparação entre os testes. Não houve diferenças estatisticamente significativas no desempenho dos alunos nos testes $-\mathrm{MH}(147)=$ $0,55, \mathrm{p}=0,68$. Dos 147 estudantes que realizaram os dois testes, 30 concordaram com o Comentário 2 nos dois. Dos 117 estudantes restantes, apenas 25 (21\%) concordaram com o Comentário 2 no pós-teste, revelando uma sofisticação em suas concepções sobre a dispersão de um conjunto de dados experimentais. Já 17 alunos (15\%) apresentaram concepções mais adequadas no pré-teste. Uma interpretação possível é a de que a realização das 13 atividades laboratoriais desenvolvidas durante o ano letivo não contribuiu e pode ter até prejudicado o desenvolvimento de concepções mais sofisticadas sobre os conceitos relacionados à média, dispersão de dados, intervalo de incerteza etc., envolvidos nessa questão. 
Tabela 5 - Comparação dos resultados dos testes - Questão 3.

\begin{tabular}{cccccc}
\hline & Comentário & Comentário 2 & $\begin{array}{c}\text { Pós-teste } \\
\text { Comentário 1 }\end{array}$ & Comentário 3 & Total \\
\hline \multirow{3}{*}{ Pré-teste } & Comentário 2 & 30 & 2 & 17 & 49 \\
& Comentário 1 & 1 & 0 & 2 & $\mathbf{3}$ \\
& Comentário 3 & 24 & 0 & 71 & $\mathbf{9 5}$ \\
\hline & Total & $\mathbf{5 5}$ & $\mathbf{2}$ & $\mathbf{9 0}$ & $\mathbf{1 4 7}$ \\
\hline
\end{tabular}

Os estudantes que optaram pelo Comentário 2 demonstram que, além da média dos valores obtidos, consideram também a dispersão do conjunto na avaliação da qualidade dos dados. É claro que a grande maioria não utilizou esse vocabulário ou esses termos, mas deixou clara sua ideia:

Os dados do grupo B são melhores, pois as medidas estão mais próximas da média e variam menos do que o grupo A que possui medidas muito distantes tanto da média quanto umas das outras.

O comentário 2, porque os melhores valores são os mais próximos entre si e da média.

A precisão do grupo B é maior pois todos valores encontrados estão próximos da média.

Esses estudantes demonstraram se orientar pelo paradigma de Conjunto, pois avaliaram os conjuntos dos dados tendo como referência não apenas o valor da média, mas levando em consideração a dispersão dos dados, reconhecendo sua importância para a avaliação da qualidade do conjunto de dados experimentais.

O resultado aqui obtido é similar aos resultados obtidos nas pesquisas de Lubben e Millar (1996) e Rollnick et al. (2001). Em ambas as pesquisas, a maioria dos estudantes também argumentou que a qualidade dos dois conjuntos de dados é a mesma, pois possuem a mesma média. Portanto, assim que aprendem a operacionalizar e a calcular a média de um conjunto de valores, os estudantes passam a considerá-la o único critério de avaliação dos dados, não considerando a dispersão do conjunto.

Coelho e Seré (1998) também obtiveram resultados semelhantes. Trabalhando com estudantes do Ensino Médio francês, as autoras concluíram que os estudantes sabem efetuar o cálculo da média de um conjunto de valores. Porém, a grande maioria dos estudantes não consegue atribuir à média seu significado e importância.

Um resultado semelhante também foi obtido por Leach et al. (2000). Nesse estudo, os autores aplicaram um questionário para mais de 400 estudantes de ensino médio e mais de 200 estudantes de Física, Química e Biologia de seis países europeus. Os autores identificaram que muitos dos estudantes, em todos os níveis investigados, sabem que devem utilizar sempre a 
média aritmética para representar o resultado final de uma série de medidas. Porém, os estudantes participantes acreditam que a média é tudo que interessa quando se compara dois conjuntos de dados, mesmo se eles apresentam intervalos de incerteza diferentes.

\section{Considerações finais}

Com as Questões 1 e 2, buscou-se identificar a evolução das concepções dos estudantes sobre a importância e o significado do cálculo da média para a determinação do valor mais representativo de um conjunto de dados experimentais. Houve uma melhora significativa no pós-teste em relação ao pré-teste. Quase dois terços dos estudantes respondentes do pós-teste reconheceram, ao final do primeiro ano do Ensino Médio que a média é o melhor valor para representar um conjunto de dados.

Mesmo tendo realizado 13 práticas ao longo do ano letivo e, na grande maioria (10 atividades) terem calculado o valor médio de diversos conjuntos de dados, ainda assim, boa parte dos estudantes do grupo pesquisado não reconhece a necessidade de se efetuar esse cálculo. Esses estudantes continuam acreditando que o valor que mais se repete dentro do conjunto (moda) é o mais indicado para representá-lo. Além disso, foram poucos os estudantes que conseguiram atribuir à média, o seu real significado e importância. Para boa parte dos estudantes pesquisados, o cálculo do valor médio é apenas um procedimento que deve ser executado muitas vezes nas atividades práticas de Física, mas não compreendem bem a razão disso.

A Questão 3 teve o objetivo de analisar se os estudantes do grupo pesquisado conseguem perceber a importância de se considerar a dispersão de dados para a avaliação da qualidade de conjuntos de dados experimentais. Realmente, não se esperava um aumento significativo da sofisticação da resposta dos estudantes para essa questão. Como foi dito, os estudantes tiveram, logo no início do ano, 3 aulas teóricas (50 minutos cada) sobre teoria de erros, tipologia de erros e cálculo de média e desvio absoluto médio e também realizam uma atividade prática que aborda diretamente esse assunto. Mas, no restante do ano, esses assuntos não foram trabalhados explicitamente ao longo das demais atividades. E, como já foi dito, na grande maioria dessas atividades, os estudantes tiveram que efetuar o cálculo do valor médio para representar uma grandeza medida, mas não calculam qualquer medida de dispersão, nem discutiram explicitamente sobre o tema. Portanto, a grande ênfase e a importância dada à média, demonstrada nas respostas dos estudantes, parecem refletir esse fato. Para a maioria dos estudantes que participaram da pesquisa, de acordo com os resultados dessa questão, se dois conjuntos de dados tiverem a mesma média, esses conjuntos são equivalentes, ou seja, têm a mesma qualidade, independentemente da dispersão dos dados de cada um.

Os resultados dessa pesquisa corroboram os resultados obtidos por Volkwy et al. (2008) ao avaliarem o impacto de um curso introdutório de Física Experimental no ensino superior. Segundo eles, praticamente todos os estudantes melhoraram seu desempenho em procedimentos mecânicos e rotinas numéricas, enquanto poucos alunos desenvolveram uma compreensão profunda dos conceitos que envolvem a análise da qualidade de dados experimentais. 
A adoção do referencial proposto por Buffler et al. (2001) ajuda-nos a definir, claramente, um dos objetivos para as atividades práticas. Esse objetivo seria o de estimular e provocar nos estudantes a mudança progressiva do paradigma Pontual para o de Conjunto (LUBARÚ; BARROS, 2009). Para isso, uma atenção maior deve ser dada, por parte dos professores, não apenas à aprendizagem dos algoritmos necessários ao tratamento dos dados, mas aos significados reais e práticos que esses construtos estatísticos possuem.

A compreensão inadequada dos estudantes sobre o significado do cálculo do valor médio e sobre a importância da dispersão dos valores para a avaliação da qualidade dos dados coletados nos leva a novos questionamentos que possibilitam novas pesquisas na área. Como os estudantes entendem a necessidade de se repetir a coleta de dados por diversas vezes? Como eles entendem a variabilidade dos dados experimentais? A que fatores eles atribuem essas variações?

As respostas para essas perguntas são importantes para que possamos identificar as concepções dos estudantes sobre o processo de medição durante a experimentação e elaborar estratégias que permitam a eles desenvolver uma compreensão mais sofisticada de todos os processos envolvidos em uma atividade experimental.

\section{Referências bibliográficas}

ALLIE, S. et al. First-year physics students' perception of the quality of experimental measurements. International Journal of Science Education, v. 20, n. 4, p. 447-459, 1998.

AMERICAN ASSOCIATION FOR THE ADVANCEMENT OF SCIENCE. Science for all americans - Project 2061. New York: Oxford University Press, 1990. 272 p.

BORGES, A. T. Novos rumos para o laboratório escolar de ciências. Caderno Catarinense de Ensino de Física, v.19, n. 3, p. 291-313, 2002.

BRASIL. Ministério da Educação. Secretaria de Educação Média e Tecnológica. Parâmetros Curriculares Nacionais: Ensino Médio. Brasília: MEC/SEMTEC, 1999. 4v.

BUFFLER, A. et al. The development of first year physics students' ideas about measurements in terms of point and set paradigms. International Journal of Science Education, v. 23, n. 11, p. 1137-1156, 2001.

COELHO, S. M.; SÉRÉ, M. G. Pupils’’ reasoning and practice during hands-on activities in the measurement phase. Research in Science \& Technological Education, v. 16, n. 1, p.79-96, 1998.

HELENE; O.; TSAI, S. P.; TEIXEIRA, R. R. P. O que é uma medida? Revista de Ensino de Física, v. 13, n. 12, p. 12-29, 1991. 
KUNG, R. L.; LINDER, C. University students' ideas about data processing and data comparison in a physics laboratory course. Nordic Studies in Science Education, v. 4, p. 40-53, 2006.

LABURÚ, C. E.; BARROS, M. A. Problemas com a compreensão de estudantes em medição: razões para a formação do paradigma Pontual. Investigações em Ensino de Ciências, v. 14, n. 2, p. 151-162, 2009.

LABURÚ, C. E.; SILVA, O. H. M.; SALES, D. R. Superações conceituais de estudantes do ensino médio em medição a partir de questionamentos de uma situação experimental problemática. Revista Brasileira de Ensino de Física, v. 32, n. 1, p. 1402, 2010.

LEACH, J. et al. Epistemological understanding in science learning: the consistency of representations across contexts. Learning and Instruction, v. 10, p. 497-527, 2000.

LEVIN, J.; FOX, J. A. Estatística para ciências humanas. São Paulo: Prentice Hall, 2004, 497p.

LIKHACHEV, V. P.; CRUZ, M. T. F.; MESA, J. Quantas medidas são necessárias para o conhecimento de uma grandeza física? Revista Brasileira de Ensino de Física, v. 22, n. 4, p. 456-462, 2000.

LOVETT, M.; SHAH, P. (Eds.). Thinking with data. Mahweh, NJ: Erlbaum, 2007.

LUBBEN, F. et al. Point and set reasoning in practical science measurement by entering university freshmen. Science Education, v. 85, n. 4, p. 311-327, 2001.

LUBBEN, F.; ALLIE, S.; BUFFLER, A. Experimental work in Science. In: Identifying potential for equitable access to tertiary level science. Springer Netherlands, 2010. p. 135-152.

LUBBEN, F.; MILLAR, R. Children's ideas about the reliability of experimental data. International Journal of Science Education, v. 18, n. 8, p. 955-968, 1996.

MARINELLI, F.; PACCA, J. L. A. Uma interpretação para dificuldades enfrentadas pelos estudantes em um laboratório didático de física. Revista Brasileira de Ensino de Física, v. 28, n. 4, p. 497-505, 2006.

MUNIER, V; MERLE, H.; BREHELIN, D. Teaching scientific measurement and uncertainty in Elementary School. International Journal of Science Education, v. 35, n. 16, p. 27522783, 2013.

NATIONAL RESEARCH COUNCIL. Inquiry and the National Science Education Standards: A Guide for Teaching and Learning. Washington, DC: National Academy Press, 2000. $224 \mathrm{p}$.

ROLLNICK, M. et al. Views of South Africa chemistry students in university bridging programs on the reliability of experimental data. Research in Science Education, v. 31, p. 553$573,2001$. 
VOLKWYN, T. S. et al. Impact of a conventional introductory laboratory course on the understanding of measurement. Physical Review ST Physics Education Research, 4, 010108, 2008.

VUOLO J. H. Fundamentos da Teoria de Erros. São Paulo: Edgard Blucher, 1996, 225p.

WHITE, R. T.; ARZI, H. J. Longitudinal studies: designs, validity, practicality, and value. Research in Science Education, v. 35, p. 137-149, 2005. 